Accepted, October 1995.

\title{
Multi-Domain Model for Pore-Size Dependent Transport of Solutes in Soils
}

\author{
W. DURNER ${ }^{1}$ and H. FLÜHLER ${ }^{2}$ \\ ${ }^{1}$ Hydrology, University of Bayreuth, 95440 Bayreuth (Germany) \\ ${ }^{2}$ Soil Physics, Institute of Terrestrial Ecology, Swiss Federal Institute of \\ Technology Zürich ETHZ, Grabenstrasse 3, 8952 Schlieren (Switzerland)
}

\begin{abstract}
Durner, W. and Flühler, H. 1995. Multi-domain model for pore-size dependent transport of solutes in soils. Geoderma, 00: 000-000.

A multi-domain model for the transport of chemicals in soils is developed. The solute flux is related to the microscopic water flux, which is modelled using concepts to estimate the hydraulic conductivity of porous media. The pore space of the soil is divided into an arbitrarily large number of domains each representing an equivalent pore radius. The domains are arranged on a structural coordinate, perpendicular to the direction of mean water flow. Transport in the flow direction takes place in each domain by convection and diffusion with pore-size specific velocities. Solute mixing between the domains is simulated as convective-dispersive transport along the structural coordinate. The model is solved numerically for one-dimensional steady-state water flux under unit-gradient conditions. Required input parameters are the unsaturated conductivity function of a soil and a pore interaction coefficient which characterizes the solute exchange between the pore domains. Simulations show a gradual change from convection dominated transport (isolated tube model) to convective-dispersive transport. The length scale where this change takes place depends on the lateral mixing intensity, pore-size distribution of the medium, and saturation degree.
\end{abstract}

\section{Introduction}

Two basically different model concepts are widely used to describe solute transport in natural soils. In the convective-dispersive approach it is assumed that the solute mass is displaced with the bulk mass of (mobile) water and is subject to dispersive spreading which can be described by Fick's law. In the stochastic approach, the soil is subdivided into isolated flow domains in which transport takes place, either by local convection-dispersion or by convection alone. 
The two process models are limiting cases for the dispersive behaviour of a solute. In the convective-dispersive model, the travel time variance grows linearly with the travel distance, whereas the stochastic convective model predicts a quadratic increase (Flühler et al., 1995). As Jury (1988) points out, it is not possible to determine the proper process model for a system from a single breakthrough experiment. Both types of models may fit a solute breakthrough equally well, but will lead to considerably different predictions for longer times. Field observations have shown that for short distances and in non-layered soils, a solute pulse tends to spread according to the stochastic-convective model (Butters et al., 1989). In soils where strong lateral mixing takes place due to small-scale heterogeneities and layering, the convective-dispersive model appears to be more appropriate (Roth et al., 1991). In a medium which is locally heterogeneous, but has distance-invariant statistical properties, we must expect a transition from stochastic-convective to convective-dispersive behavior with increasing transport distance (Jury and Flühler, 1992; Russo et al., 1994).

Observations of preferential flow have lead to modifications of the convection-dispersion model (two-region concepts and multi-region concepts). However, many natural soils do not consist of two or three distinct domains. Therefore, the parameters of such models depend on the water content. This makes their determination for transient conditions very laborious and limits the use of these models for practical purposes.

Obviously, some of the limitations of the above approaches could be overcome by considering the detailed velocity structure of the carrier fluid in the porous medium to estimate the transport properties. However, it is not possible to deterministically model the threedimensional transport on pore-scale, since the properties are neither known, nor observable, nor predictable with currently available methods. Further, the computational requirements were so high that the modeling efforts were impractical.

Some attempts have been made to use the statistical information on the local flow velocity, as obtained from the pore-size distribution and hydraulic conductivity function of a soil. Lindstrom and Boersma (1971) separated the pore space of a saturated soil into a finite number of sub-domains with characteristic pore sizes. They estimated the flow velocity in each pore domain by Poiseuille's law and calculated total transport by superimposing analytical solutions of the convection-dispersion equation. Their model was not successful in describing experimental breakthrough curves (Rao et al., 1976) since it did not include any mixing of solutes between the pore classes.

To describe solute transport due to preferential flow in unsaturated soils, Steenhuis et al. (1990) presented a model where a piecewise linear approximation of the macroscopic hydraulic conductivity function was used to derive the convection velocities in groups of different pore sizes. Lateral solute exchange between the groups was modeled for each depth-layer by mixing the solutes of a certain fraction of each pore group in a virtual mixing cell. By choosing the velocities of the pore groups to be an integer multiple of the adjoining group, a simple numerical solution could be obtained. However, this constraint limits the maximum number of pore groups and leads, therefore, to a very ragged resolution.

Recently, Skopp and Gardner (1992) and Durner (1992) presented an analytical model for a continuous distribution of local flow velocities on an axis orthogonal to the main transport direction. Transport in each domain takes place by convection and dispersion. Solute exchange between the domains is modeled as a diffusion-like process along this axis. Skopp and Gardner (1992) solved the model for one-dimensional saturated transport with the method of moments. They showed that the macroscopic dispersion coefficient increases with the microscopic flow velocity variance and linearly depends on the average flow velocity. For large distances, they showed the equivalence of the model with the classical convection-dispersion approach. These findings are analogous to the theory of Taylor dispersion on the scale of a single tube (Taylor, 1953) and to results from solute transport modelling in layered aquifers (Matheron and de Marsily, 1980; Berkowitz et al., 1988) on the macroscopic scale.

In this paper, we generalize the multi-domain approach for non-stationary unsaturated so- 
lute transport. The model is solved numerically for a one-dimensional case under simplified hydraulic conditions. The number of pore classes can be chosen arbitrarily, and mixing is treated as a dispersion-like process along a coordinate oriented perpendicular to the direction of water flow. The definition of such a coordinate in terms of pore-size distribution and allowing mixing on this coordinate leads to a very flexible model which describes naturally occurring transport patterns, from strongly preferential to convective-dispersive transport including the transition between the two extremes - with a minimum number of unique parameters.

The paper is arranged into three parts. First, we will present the basic theory which results in the statement of the governing differential equations. Second, a numerical solution for a simplified case is outlined. Finally, the numerical model is applied to demonstrate some basic properties of the approach.

\section{Theory}

On a microscale, water and chemical transport takes place in a highly complex three-dimensional pore space which, in practice, cannot be described phenomenologically. On the observation scale, the properties of the medium are described by the continuum approach, which assigns lumped variables such as water content and hydraulic potential to each point in space. The description for saturated/unsaturated water transport is given by Richards' equation, which attributes to every point in space, $\mathbf{x}$, a water flow velocity vector, $v$. This macroscopic velocity is usually used to calculate the convective movement of solutes.

Following the statistical pore-bundle concepts that have been developed to estimate the unsaturated hydraulic conductivity (Childs and Collis-George, 1953; Burdine, 1953; Mualem, 1976) we can represent the pore-space as a network of capillaries with a frequency distribution of diameters equivalent to the pore-size distribution of the porous medium. This distribution is deduced from the water retention curve by means of the capillary law, which relates the energy status at a given water content to an equivalent pore size. By integrating the fluxes of the water-filled cross-section, the macroscopic hydraulic conductivity is estimated. Despite the physically unrealistic abstraction of pore geometries, this concept can be used to estimate unsaturated water flux densities in porous media (Mualem and Dagan, 1978; Mualem, 1992).

An attempt to model the transport of solutes in a direct analogy to the calculation of the hydraulic conductivity will not be successful, because "small" and "large" pores are generally not spatially isolated voids, but result from different filling degrees of a continuous pore space (Fig. 1). We, therefore, treat regions of pores with different sizes not as spatially isolated capillaries, but as overlaying continua. To model the mixing of solutes between these continua, we define a virtual coordinate, $\xi(-)$, which will be used as a measure of distance. It is oriented orthogonal to the direction of the macroscopic water flux, and has no geometric interpretation. In order to clearly distinguish this coordinate from the cartesian ones, we will subsequently call it a structural coordinate. All the pore sub-domains are arranged along the structural coordinate in an ascending order according to their pore sizes. It is convenient to choose the coordinate to be numerically identical to the water content at which pores of a certain size fill or drain. By this, pores of similar size are in close neighborhood, whereas the smallest and the largest pores are farthest apart. This conceptualization of "neighborhood" of different pore regions is, of course, not the only possible one, but has to be regarded as one extreme of two opposing possibilities. In the model of Steenhuis et al. (1990), e.g., all pore classes are in equal interaction. A weighed mixture of both concepts were most flexible and probably would lead to the best results when compared to real soils. However, the combination of both concepts would increase the number of fitting coefficients in the model and is not considered in this paper. 


\section{Modeling of the Water Flux}

The macroscopic water flux density vector, $q(\mathbf{x})\left(\mathrm{LT}^{-1}\right)$ in a saturated or unsaturated soil can be decomposed at any spatial position, $\mathbf{x}$, into a continuous distribution of pore-size specific (differential) flux densities, $q^{\prime}(\mathbf{x}, \xi)\left(\mathrm{LT}^{-1}\right)$, directed in the same direction as $q$

$$
q(\mathbf{x})=\int_{0}^{\theta(\mathbf{x})} q^{\prime}(\mathbf{x}, \xi) d \xi
$$

The structural coordinate ranges from zero to the saturated water content, $\theta_{s}$. The integration covers all water filled pores from water content 0 to $\theta(\mathrm{x})\left(\mathrm{L}^{3} \mathrm{~L}^{-3}\right)$, the volumetric water content at point $\mathrm{x}$. Note that the differential flux components, $q^{\prime}$, are equal to the domain-specific pore water velocity components, $v(\xi)$, in the macroscopic flow direction. To determine these velocities, let us assume that the macroscopic gradient of the hydraulic potential, $\nabla \phi$, acts equally on pores of arbitrary size, that Darcy's law is valid in each pore domain, and that hysteresis of the unsaturated hydraulic conductivity function, $K(\theta)$, can be neglected. The Darcy law

$$
q=\bar{v} \theta=K \nabla \phi
$$

where $\bar{v}$ indicates the average pore water velocity, can be decomposed to

$$
q=\int_{0}^{\theta} v(\xi) d \xi=\int_{0}^{\theta} K^{\prime}(\xi) d \xi \nabla \phi
$$

where $K^{\prime}\left(\mathrm{LT}^{-1}\right)$ is the hydraulic conductivity of the pore domain at $\xi$. From Eq. (2) and Eq. (3) it follows that

$$
v(\xi)=K^{\prime}(\xi) \nabla \phi=\left.\frac{\partial K}{\partial \theta}\right|_{\xi} \nabla \phi
$$

This means that the flow velocity distribution over the various pore domains, $\xi$, is given by the derivative of the macroscopic conductivity function. By this concept, water moves faster through large pores than through fine pores. When water infiltrates into dry soil it has to move at the infiltration front from large pores to fine pores. This is a local redistribution of water on the structural coordinate which can be modeled by considering a flux component along the structural axis. The Richards equation requires that this local redistribution of water is an instantaneous process, i.e., that at any point in space, the smaller pores will always be water-filled. If the process of water redistribution along the structural coordinate is explicitly taken into account and treated as a slow process, bypass flow in macropores is modeled.

\section{Modeling Chemical Transport}

The transport of a conservative chemical in a porous medium (in dissolved, colloidal, or particle-adsorbed phase) is caused by two physical processes: (i) advective transport in the mobile fluid phase, and (ii) molecular diffusion. To model the macroscopic dispersion process which results from different flow velocities of the carrier fluid, we divide the total solute flux into pore-size specific differential fluxes. According to the conceptual model for the water flux, this solute flux distribution lies on a structural coordinate orthogonal to the direction of water flow. Transport in the main flow direction is modeled by convective-diffusive transport within each pore domain, and exchange of solutes between the different pore domains by convective-diffusive transport on the structural coordinate. Convection in the main flow direction takes place with a pore-size specific flow velocity distribution, $v(\xi)$ Eq. (4). Accordingly, the solute flux is

$$
J(\mathbf{x}, \xi)=v(\mathbf{x}, \xi) c(\mathbf{x}, \xi)-\tau D_{o} \nabla c(\mathbf{x}, \xi)
$$


where $J\left(\mathrm{ML}^{-2} \mathrm{~T}^{-1}\right)$ is the domain specific solute flux in the macroscopic flow direction, $c$ $\left(\mathrm{ML}^{-3}\right)$ is the pore-size specific concentration at $\mathbf{x}, D_{\circ}\left(\mathrm{L}^{2} \mathrm{~T}^{-1}\right)$ is the molecular diffusion coefficient of the solute in water, and $\tau(-)$ is a tortuosity factor. As long as transport velocities are not very small, the longitudinal spreading due to molecular diffusion is negligible as compared to the spreading caused by the velocity differences between the flow domains and the second term in Eq. (5) may be ignored.

The macroscopic resident concentration, $\bar{c}^{r}$, and the macroscopic flux concentration, $\bar{c}^{f}$, are obtained by integration

$$
\begin{gathered}
\bar{c}^{r}=\frac{1}{\theta} \int_{0}^{\theta} c(\xi) d \xi, \\
\bar{c}^{f}=\frac{1}{q} \int_{0}^{\theta} c(\xi) v(\xi) d \xi .
\end{gathered}
$$

The intensity of lateral solute exchange between the pore domains depends on the convective movement from large to small pores and on the dispersive lateral mixing determined by two basic properties of the porous medium: (i) spatial arrangement, tortuosity, and continuity of the pores, and (ii) a characteristic mixing length, expressing the length scale of the microscopic heterogeneities of the porous medium. The mixing length scale can be explicitly included in the model by scaling the structural coordinate. However, it is not possible to determine the two properties independently from morphological and soil physical measurements. Therefore, we lump both properties into a domain interaction coefficient, $\alpha_{\xi}\left(\mathrm{L}^{-1}\right)$, and simulate the solute flux between the pore domains by

$$
\mathbf{J}_{\xi}(\mathbf{x})=w(\mathbf{x}, \xi) c(\mathbf{x}, \xi)-\alpha_{\xi} \frac{\partial c(\mathbf{x}, \xi)}{\partial \xi}
$$

where $\mathbf{J}_{\xi}(\mathbf{x})\left(\mathrm{MT}^{-1}\right)$ is the solute flux on the structural coordinate at $\mathbf{x}, w\left(\mathrm{~T}^{-1}\right)$ is the convective water flux on the structural axis, and $\alpha_{\xi}$ is the domain interaction coefficient, as defined above. In general, $\alpha_{\xi}$ may depend on $\xi$ as well as on the pore water velocity. Equation (8) is similar in form to that given by Gerke and van Genuchten (1993) for transport in dual-porosity systems.

The complete transport equation for the pore-size specific concentrations, $c$, is

$$
\frac{\partial c}{\partial t}=-\nabla\left(v c-\tau D_{o} \nabla c\right)-\frac{\partial}{\partial \xi}\left(w c-\alpha_{\xi} \frac{\partial c}{\partial \xi}\right) .
$$

where $v, c$, and $w$ are functions of the spatial and the structural position.

Equation (9) must be solved for appropriate initial and spatial boundary conditions. This is done by solving the Richards equation and calculating $v$ and $w$ for each spatial and structural position. This flow field can be coupled with a solute transport simulation scheme. Since an additional structural coordinate is used, the dimensionality of the problem is increased. One-dimensional solute transport can be modeled with a two-dimensional scheme, twodimensional solute transport with a three-dimensional scheme. A fully three-dimensional transport problem would require a four-dimensional scheme (to the authors' knowledge, in the field of hydrology or soil physics there are, at present, no simulation codes for this problem available). Because the problem is advection dominated, numerical dispersion will be a major problem when applying finite-element or finite-difference techniques. A particle tracking scheme may be a more appropriate solution method.

When Eq. (9) is solved, the macroscopic solute flux is obtained by integrating the domainspecific fluxes

$$
\mathbf{J}(\mathbf{x})=\int_{0}^{\theta(\mathbf{x})}\left\{v(\mathbf{x}, \xi) c(\mathbf{x}, \xi)-D_{\circ} \nabla c(\mathbf{x}, \xi)\right\} d \xi .
$$




\section{Simplified Problem}

In the following section, we will outline a numerical model for the simplified case of onedimensional transport of a solute pulse through a column of finite length under unit-gradient conditions. By assuming this, the lateral convective transport component will be zero and no explicit microscopic simulation of the water flow is required. We further assume that the pore interaction factor, $\alpha_{\xi}$, is independent of the spatial and structural position and neglect molecular diffusion. The governing differential equation for this problem is given by

$$
\frac{\partial c}{\partial t}=-v \frac{\partial c}{\partial z}+\alpha_{\xi} \frac{\partial^{2} c}{\partial \xi^{2}}
$$

The initial condition is

$$
c(z, \xi, t)=0 \quad \forall z \text { and } \forall \xi ; \quad t \leq 0 .
$$

The boundary conditions are

$$
\begin{aligned}
& c\left(z_{o}, \xi, t\right)=\left\{\begin{array}{lll}
c_{o} & \text { for } 0 \leq \xi \leq \theta\left(z_{o}\right) ; & 0 \leq t \leq t_{0} \\
0 & \text { for } 0 \leq \xi \leq \theta\left(z_{o}\right) ; & t \geq t_{0}
\end{array} .\right. \\
& c\left(z_{L}, \xi, t\right)=0 \quad \text { for } 0 \leq \xi \leq \theta\left(z_{L}\right) ; \quad t \geq 0
\end{aligned}
$$

where $z_{0}$ indicates the soil surface and $z_{L}$ the lower boundary.

On the structural axis, the boundary conditions for both water and chemical transport are given by the no-flux requirement at $\xi=0$ and $\xi=\theta(z)$.

Note that in contrast to the macroscopic convection-dispersion models, a flux-type boundary condition can be transformed to the first-type boundary condition, Eq. (13), by simply dividing the solute flux by the water flux. Since macroscopic dispersion is simulated by local solute fluxes and molecular diffusion is neglected, no backdispersion occurs across the boundaries.

The simplified model, represented by Eqs. (11) - (14) suffers from assumptions which are only approximately met in nature, the most important being the the unit gradient assumption. While this situation is quite common in the field after extended infiltration, it is certainly not true at infiltration fronts and during periods of significant evaporation. Nevertheless, the simplified model will clarify the basic features of the approach.

\section{Numerical Model}

In the following, we describe a numerical scheme for the simulation of one-dimensional saturated/unsaturated multi-domain solute transport under steady-state unit-gradient conditions ${ }^{1}$. Following the method of Steenhuis et al. (1990), we apply a two-step procedure for convective transport and mixing. In the first step, the transport in each pore domain along the spatial axis is solved. In the second step, solute exchange between the pore domains is modelled by a finite difference approximation of the diffusion equation.

\footnotetext{
${ }^{1}$ Details of the numerical treatment are documented in an internal report (Durner W. 1995. Multi-Domain Model for Transport of Chemicals in Structured Soils. Report 95.2. Department of Hydrology, University of Bayreuth, Germany). The simulation program and the report are available from the senior author.
} 


\section{Numerical Discretization}

The soil depth from $z=z_{0}$ at the surface to $z=z_{L}$ at the lower boundary is divided into $n_{l}$ layers (or compartments) of equal length, $\Delta z$. The mass of each layer is lumped at the central nodal position $z_{i}=z_{0}+\left(i-\frac{1}{2}\right) \Delta z, i=1, \ldots, n_{l}$. The total pore space, $\epsilon$, is divided into $n_{c}$ pore classes that cover the whole range of pore sizes. The pore classes are arranged along the structural coordinate, $\xi$, according to their equivalent pore diameters. To simplify the notation, we assume here that each pore class occupies an equal volumetric fraction, $\Delta \epsilon=\epsilon / n_{c}$. The $j$-th pore class, therefore, ranges from water content $\theta_{j-1}=(j-1) \Delta \epsilon$ (when the pore class is fully drained) to $\theta_{j}=j \Delta \epsilon$ (when it is filled). On the structural coordinate, the water content and solute mass of each pore class is averaged and assigned to position $\xi=\frac{1}{2}\left(\theta_{j}+\theta_{j-1}\right), j=1, \ldots, n_{c}$.

By this discretization, the soil is subdivided into $n_{l} \times n_{c}$ subdomains that will be referred to as cells $(i, j)$. Each cell is characterized by its spatial and structural position, by its water saturation $s_{i j}(0 \leq s \leq 1)$, and its solute concentration, $c_{i j}$, where the index $i$ indicates the depth layer and $j$ indicates the pore class.

The macroscopic properties water content, $\theta\left(z_{i}\right)$, water flux, $q\left(z_{i}\right)$, volume averaged concentration, $\bar{c}^{r}\left(z_{i}\right)$, and flux-averaged concentrations, $\bar{c}^{f}\left(z_{i}\right)$, at depth $z_{i}$ are obtained by summation over all pore classes:

$$
\begin{gathered}
\theta\left(z_{i}\right)=\sum_{j=1}^{n_{c}} \Delta \epsilon s_{i j}, \\
q\left(z_{i}\right)=\sum_{j=1}^{n_{c}} v_{j} \Delta \epsilon s_{i j}, \\
\bar{c}_{\left(z_{i}\right)}^{r}=\frac{1}{\theta\left(z_{i}\right)} \sum_{j=1}^{n_{c}} \Delta \epsilon s_{i j} c_{i j}, \\
\bar{c}_{\left(z_{i}\right)}^{f}=\frac{1}{q\left(z_{i}\right)} \sum_{j=1}^{n_{c}} v_{j} \Delta \epsilon s_{i j} c_{i j} .
\end{gathered}
$$

\section{Vertical Transport}

The water flux at depth $z_{i}$ is a sum of the fluxes in the $n_{c}$ pore classes

$$
q\left(z_{i}\right)=\sum_{j=1}^{n_{c}} \Delta q_{j}\left(z_{i}\right) .
$$

The soil solution is displaced in the different pore classes with characteristic average velocities, which are - within a pore class - constant over depth. The water flux in pore class $j, \Delta q_{j}$, is under unit-gradient conditions equal to the increase in the hydraulic conductivity, $\Delta K(\theta)$, which is caused by the pores of that class

$$
\Delta q_{j}=K\left(\theta_{j}\right)-K\left(\theta_{j-1}\right)
$$

The water flow velocity, $v_{j}$, in that class is accordingly

$$
v_{j}=\frac{K\left(\theta_{j}\right)-K\left(\theta_{j-1}\right)}{\theta_{j}-\theta_{j-1}} .
$$

Vertical solute transport is simulated with the method of characteristic, extending the solution scheme used by Steenhuis et al. (1990). During each timestep, infiltrating water and chemical mass are temporarily stored in pore-size specific surface cells. After the timestep, a 
decision is made for each pore class whether the content of the surface cell is moved into the first layer in the soil, a process called propagation. If a propagation occurs, the contents of all cells of the pore class are moved simultaneously to the next layer. The content of the bottom cell is pushed out of the soil domain and is used to calculate the lower boundary fluxes. The mechanism is explained in Fig. 2.

The timestep, $\Delta t$, in a simulation depends on the spatial discretization, $\Delta z$, and on the largest value of the pore water velocities, $v_{\text {max }}$, according to

$$
\Delta t=\Delta z / v_{\max }
$$

where $v_{\max }$ is the velocity of the class with the largest water-filled pores. Accordingly, the content of this pore class propagates exactly the distance $\Delta z$ during one timestep.

The numerical solution is exact for all pore classes if their velocities are integer multiples of each other. Unfortunatley, this requirement severely restricts the freedom to choose the number and ranges of pore classes. Simulations with small numbers of pore classes lead to ragged breakthrough curves (see Results section). Therefore, we introduce a virtual storage compartment at the soil surface by which the propagation scheme is applicable for classes with arbitrary velocities (Fig. 2). After each timestep and for each class, a decision is made whether a propagation takes place or not. No propagation will occur if the pore-group specific accumulated (virtual) flow distance, $x_{j}^{v i r t}$, which is at the end of the $k$-th timestep given by

$$
x_{j}^{v i r t}=\sum_{\nu=1}^{k} v_{j} \Delta t \quad
$$

is smaller than the already simulated propagation distance at the beginning of that timestep, $x_{j}$ (which is an integer multiple of $\Delta z$ ) plus one nodal distance. If $x_{j}^{v i r t} \geq x_{j}+\Delta z$, a propagation is performed, and $x_{j}$ is increased by $\Delta z$. By this scheme, any number of pore classes with arbitrary ranges on the structural coordinate can be chosen, which greatly enhances the flexibility of the method. However, the numerical scheme is now slightly biased by retarding the input of the chemical at the upper boundary to the system.

\section{Mixing}

The dispersive mass exchange on the structural coordinate is modelled after each timestep by a fully implicit finite difference approximation of the second term of the right-hand side Eq. (11), with no-flux boundaries at $\xi=0$ and $\xi=\theta\left(z_{i}\right)$. In our examples, the interaction coefficient, $\alpha_{\xi}$, is assumed to be independent of the pore size. In the more general case, we may express $\alpha_{\xi}$ as a function of $\xi$. By attributing different exchange intensities to different pore sizes, bypass-transport can be simulated.

\section{Initial and Boundary Conditions}

At the beginning of the simulation, the water saturations of all cells are set to an initial value, which is equal to the water content at the upper boundary (which, in turn, may be determined by the applied flux rate or the matric potential). Due to the steady-state and unitgradient condition, the lower boundary is set to an identical value. For chemical transport, the upper boundary condition is determined by the concentration, $c_{s u r f}(t)$ in the infiltrating water, which is equal for all pore classes. As discussed, flux and concentration boundary conditions are related to each other by $c_{\text {surf }}=\mathbf{J} / q$, because no longitudinal dispersion occurs within a pore class. At the lower boundary, the mass flux is calculated by summing up the contents of those cells which have left the soil domain during a timestep. 


\section{Additional Processes}

Steenhuis et al. (1990) have shown for their model that the incorporation of additional processes like water extraction through plant roots and degradation of chemicals is straightforward. Of particular interest is the simulation of transient processes. If infiltration into a dry soil is simulated, the propagation of water in the larger pores is faster than in the smaller ones, leading to depth zones where large pores are water filled, whereas small ones are not. When this situation occurs, the water in the larger pores moves on the structural coordinate into the smaller ones. This may be treated as an instantaneous or a kinetically controlled process. Since we want to stress in this paper the basic features of the multi-domain approach, we have restricted this presentation to the transport of an ideal tracer under the simplest possible hydraulic regime.

\section{Results}

In order to demonstrate the continuous change between strongly preferential transport and convective-dispersive transport, we show simulation results for a strongly aggregated soil, Rideau Clay Loam (De Jong et al., 1992). Figure 3 (left) depicts the retention data, a fitted multimodal retention curve, and the corresponding pore-size distribution. The soil has a distinct secondary pore system. The effect of this pore system on the estimated hydraulic conductivity function is shown on the right hand side of Fig. 3. Beginning from saturation, a change of few percent of water leads to a very steep drop of the conductivity (Durner, 1994). The soil, therefore, exhibits a hydraulic characteristic which is typical for soils where preferential water and solute transport take place.

Figures 4 to 8 show simulated breakthrough curves from pulse applications of an inert tracer. The input pulse has a concentration of 100 (arbitrary unit) and a pulse length of 0.2 pore volumes. The soil column has a height of $10 \mathrm{~cm}$, which has been discretized into 40 layers. The saturated hydraulic conductivity was $100 \mathrm{~cm} \mathrm{~d}^{-1}$. The figures show the influence of varying the number of pore classes (Fig. 4), varying the interaction coefficient $\alpha_{\xi}$ (Fig. 5), varying the length of the soil column (Fig. 6), and varying the degree of saturation (Fig. 7). Finally, the influence of different pore-size distributions is shown (Fig. 8).

Figure 4 shows for the (unrealistic) case of no mixing between the pore classes, how the number of pore classes used in the simulation affects the shape of the breakthrough curve. If the entire pore space is divided into ten pore classes, the input pulse travels in 10 separate parcels downstream. Most of the total mass transfer takes part in the pore groups containing the largest pores (dashed line). Dividing the pore space into 33 pore classes yields a continuous, but still unacceptable, rough breakthrough curve (dotted line). Modeling the breakthrough curve with 100 pore classes (solid line) yields a continuous curve, with an early breakthrough and extensive tailing. This example shows that for a very small pore interaction it is necessary to discretize the structural coordinate in small intervals. If $\alpha_{\xi}$ becomes larger, the breakthrough curve is smoothed and the number of pore classes can be considerably reduced.

The influence of varying the domain interaction coefficient, $\alpha_{\xi}$, is shown in Fig. 5. The domain interaction coefficient is expressed as relative value, $\alpha_{\xi}^{*}\left(\mathrm{~L}^{-1}\right)$, which is normalized by the saturated hydraulic conductivity, $K_{s}$, according to $\alpha_{\xi}^{*}=\alpha_{\xi} / K_{s}$. A value of $\alpha_{\xi}=0 \quad$ Fig. 5 $\mathrm{cm}^{-1}$ indicates no exchange between pores of different sizes. As has been shown in Fig. 4, this physically unrealistic case leads to a very early breakthrough. The other extreme case is a complete mixing between the pores which yields a breakthrough curve identical to a convection-dispersion equation solution (dots).

Figure 6 shows the breakthrough curves for different transport distances. For a column Fig. 6 
length too short to allow complete mixing between the different flow domains, the breakthrough curve is asymmetric, indicating preferential transport. With larger transport distance, the shape approaches the typical bell-shaped curve of convective-dispersive transport. As predicted by the analytical solution of Skopp and Gardner (1992), the variance of the peak finally grows linearly with the transport distance.

Figure 7 depicts one of the most interesting results of the multi-domain approach: the transport model reflects the structure of the pore space under different flow conditions. In the saturated case the fast flux of water and solutes through only a few percent of the pore space causes a very early breakthrough of the applied solute (as may be expected in soils with preferential transport). At a lower percolation rate, where the soil is unsaturated, the situation changes dramatically. At a saturation degree of 0.8 , the flow rate is more than four orders of magnitude reduced, as compared to complete saturation (Fig. 3). The exchange process between the pore domains is now fast enough to lead to a complete mixing, which results in a typical convection-dispersion behavior. For a soil without a secondary pore system, the influence of varying the saturation degree will be similar by tendency, but less pronounced.

In the last example, transport patterns that result from different hydraulic properties are compared (Fig. 8). Three hydraulic conductivity curves have been used which are characterized by a different coefficient, $n$, of the unimodal van Genuchten/Mualem model (van Genuchten, 1980). This parameter fully determines the shape of $K(\theta)$. For saturated transport through a soil column of $20 \mathrm{~cm}$ length, the wide pore-size distribution, $(n=1.2$, characterizing a loam) releases an asymmetric transport breakthrough curve with an early arrival of the peak concentration and a considerable tailing, whereas in the soil with the more narrow pore-size distribution ( $n=3.0$, characterizing a sandy soil) the state of complete mixing has already been reached.

\section{Conclusions}

We presented a conceptual multi-domain model for the transport of solutes in variably saturated soils. The concept divides the pore space into a continuous distribution of pore-size specific continua which are distributed on a virtual structural coordinate, orthogonal to the spatial coordinate. Transport is simulated as a convective-dispersive process in the simulation space which consists of the spatial and, additionally, the structural dimension.

A one-dimensional numerical model has been used to simulate the transport of a solute pulse through soil columns under unit-gradient conditions. The simulations show a gradual change of convection-dominated transport to convective-dispersive transport. The length scale where this change takes place depends on the pore-size distribution of the porous medium, on the intensity of lateral mixing, and on the degree of saturation. The transition to convective-dispersive transport is slow if a soil has a wide pore-size distribution, a small lateral mixing intensity, and a high water saturation. Because the numerical model is subject to simplifying assumptions which are not valid in nature, it is at present not intended for practical use in field situations, but aims on basic research, validation studies, and education.

We believe that the consideration of a continuous distribution of pore-sizes in transport simulations can help to overcome some of the limits that are associated with the classical macroscopic concepts. For the future, it appears particularly interesting to apply multidomain models to transient flow regimes under physical non-equilibrium situations, such as bypass flow, and to simulate explicitly pore-size dependent physico-chemical processes, such as pore-size dependent sorption or the transport of colloides which takes place only in pores of a certain minimum size. 


\section{References}

Berkowitz, B., Bear, J. and Braester, C., 1988. Continuum models for contaminant transport in fractured porous formations. Water Resour. Res., 24(8): 1225-1236.

Burdine, N. T., 1953. Relative permeability calculations from pore size distribution data. Petrol. Trans., Am. Inst. Min. Eng., 198: 71-78.

Butters, G. L., Jury, W. A. and Ernst, F. F., 1989. Field scale transport of bromide in an unsaturated soil. 1. Experimental methodology and results. Water Resour. Res., 25(7): 15751581.

Childs, E. C. and Collis-George, N., 1953. The permeability of porous media. Proc. Roy. Soc. A, 201: 392-405.

De Jong, R., Topp, G. C. and Reynolds, W. D., 1992. The use of measured and estimated hydraulic properties in the simulation of soil water content profiles: a case study. In: M. T. van Genuchten, F. J. Leij and L. J. Lund (Editors), Proceedings of the International Workshop, Indirect Methods for Estimating the Hydraulic Properties of Unsaturated Soils. University of California, Riverside, CA, pp. 594-584.

Durner, W., 1992. New concepts for the functional description of soil hydraulic properties and for the modeling of pollutant transport during stormwater infiltration into multiporosity soils. In: M. Grottger and W. Schilling (Editors), Hydrological and Pollutional Aspects of Stormwater Infiltration. Proceedings of the European Workshop. Schriftenreihe der EAWAG, 3. EAWAG, CH-8600 Dübendorf, pp. 125-143.

Durner, W., 1994. Hydraulic conductivity estimation for soils with heterogeneous pore systems. Water Resour. Res., 30: 211-223.

Flühler, H., Durner, W. and Flury, M., 1995. Lateral solute mixing processes-A key for understanding field-scale transport of water and solutes. Geoderma, this issue: 000-000.

Gerke, H. H. and van Genuchten, M. T., 1993. A dual-porosity model for simulating the preferential movement of water and solutes in structured porous media. Water Resour. Res., 29(2): 305-319.

Jury, W., 1988. Solute transport and dispersion. In: W. Steffen and O. Denmead (Editors), Flow and Transport in the Natural Environment: Advances and Applications. Springer, New York, pp. 1-16.

Jury, W. A. and Flühler, H., 1992. Transport of chemicals through soil: Mechanisms, models, and field applications. Adv. Agronomy, 47: 141-201.

Lindstrom, F. T. and Boersma, L., 1971. A theory on the mass transport of previously distributed chemicals in a water saturated sorbing porous medium. Soil Sci., 111(3): 192199.

Matheron, G. and de Marsily, G., 1980. Is transport in porous media always diffusive? A counterexample. Water Resour. Res., 16(5): 911-917.

Mualem, Y., 1976. A new model for predicting the hydraulic conductivity of unsaturated porous media. Water Resour. Res., 12: 513-522. 
Mualem, Y., 1992. Modeling the hydraulic conductivity of unsaturated porous media. In: M. T. van Genuchten, F. J. Leij and L. J. Lund (Editors), Proceedings of the International Workshop, Indirect Methods for Estimating the Hydraulic Properties of Unsaturated Soils. University of California, Riverside, CA, pp. 15-36.

Mualem, Y. and Dagan, G., 1978. Hydraulic conductivity of soils: unified approach to the statistical models. Soil Sci. Soc. Am. J., 42: 392-395.

Rao, P. S. C., Green, R. E., Ahuja, L. R. and Davidson, J., 1976. Evaluation of a capillary bundle model for describing solute dispersion in aggregated soils. Soil Sci. Soc. Am. J., 40: 815-820.

Roth, K., Jury, W. A., Flühler, H. and Attinger, W., 1991. Transport of chloride through an unsaturated field soil. Water Resour. Res., 27(10): 2533-2541.

Russo, D., Zaidel, J. and Laufer, A., 1994. Stochastic analysis of solute transport in partially saturated heterogeneous soil. I. Numerical experiments. Water Resour. Res., 30(3): 769_ 779.

Skopp, J. and Gardner, W. R., 1992. Miscible displacement: An interacting flow region model. Soil Sci. Soc. Am. J., 56: 1680-1686.

Steenhuis, T. S., Parlange, J.-Y. and Andreini, M. S., 1990. A numerical model for preferential solute movement in structured soils. Geoderma, 46: 193-208.

Taylor, G. I., 1953. Dispersion of soluble matter in solvent flowing through a tube. Proc. R. Soc. Series A, 219: 186-203.

van Genuchten, M. T., 1980. A closed-form equation for predicting the hydraulic conductivity of unsaturated soils. Soil Sci. Soc. Am. J., 44: 473-480. 


\section{FIGURE CAPTIONS}

Fig. 1: Schematic visualization of two geometrical concepts for the pore space. (a) Pores of different sizes are spatially isolated holes in a solid matrix. The probability of pores of different sizes to be connected to each other is given by the product of their areal fraction at a cross-section (concept of Childs and Collis-George (1953)). (b) The pore space desaturates gradually when the suction increases. The grey shadings represent different degrees of desaturation. Pores of similar "size" are in closer contact to each other than pores of very different sizes.

Fig. 2: Eight timesteps of the propagation of a chemical into a water saturated soil. Each plot shows the cell arrangement for five pore classes (numbered in the lowest layer), and twelve depth layers. The relative velocities of the pore classes are $0.125,0.25$, $0.5,0.75$, and 1 , for pore classes 1 to 5 , respectively. White color indicates cells with concentration zero, grey shading indicates cells with relative concentration one. Exchange between the pore domains is zero (isolated tube model). The bars show the virtual propagation way in each pore class. As soon as the virtual propagation way is longer than the already propagated distance plus one layer thickness, a propagation step occurs. If no propagation occurs, the infiltrating chemical mass is stored in the surface layer (top of the cell array).

Fig. 3: Hydraulic properties of Rideau Clay Loam. Left: measured water retention data (dots), fitted bimodal retention curve (solid line), and underlying pore-size distribution (dark shaded, cf. Durner, 1994). Right: predicted functions $K(\psi)$ (dashed, related to top axis), and $K(\theta)$ (solid, related to bottom axis).

Fig. 4: Simulated steady-state breakthrough of a solute pulse of 0.2 pore volumes, applied to a $10 \mathrm{~cm}$ column of Rideau Clay Loam at $45 \%$ water content. Influence of varying the number of pore classes, $n_{c}$. No mixing between the pore domains is assumed.

Fig. 5: Simulated steady-state breakthrough of a solute pulse of 0.2 pore volumes, applied to a $10 \mathrm{~cm}$ column of Rideau Clay Loam at $45 \%$ water content. Influence of varying the pore-domain interaction coefficient, $\alpha_{\xi}$.

Fig. 6: Simulated breakthrough of a solute pulse of 0.2 pore volumes, applied to a column of Rideau Clay Loam at $45 \%$ water content. Normalized pore-domain interaction coefficient is set to $\alpha_{\xi}^{*}=10^{-2}$. Influence of varying the column length.

Fig. 7: Simulated breakthrough of a solute pulse of 0.2 pore volumes, applied to a $10 \mathrm{~cm}$ column of Rideau Clay Loam. Normalized pore-domain interaction coefficient is set to $\alpha_{\xi}^{*}=10^{-2}$. Influence of varying water saturation, $s$.

Fig. 8: breakthrough curves through a saturated soil column of $20 \mathrm{~cm}$ length, comparing different pore-size distribution. The soil hydraulic properties are characterized by three different values of the parameter $n$ of the van Genuchten/Mualem hydraulic model (van Genuchten, 1980). Assumed $\alpha_{\xi}^{*}=10^{-2}$. 

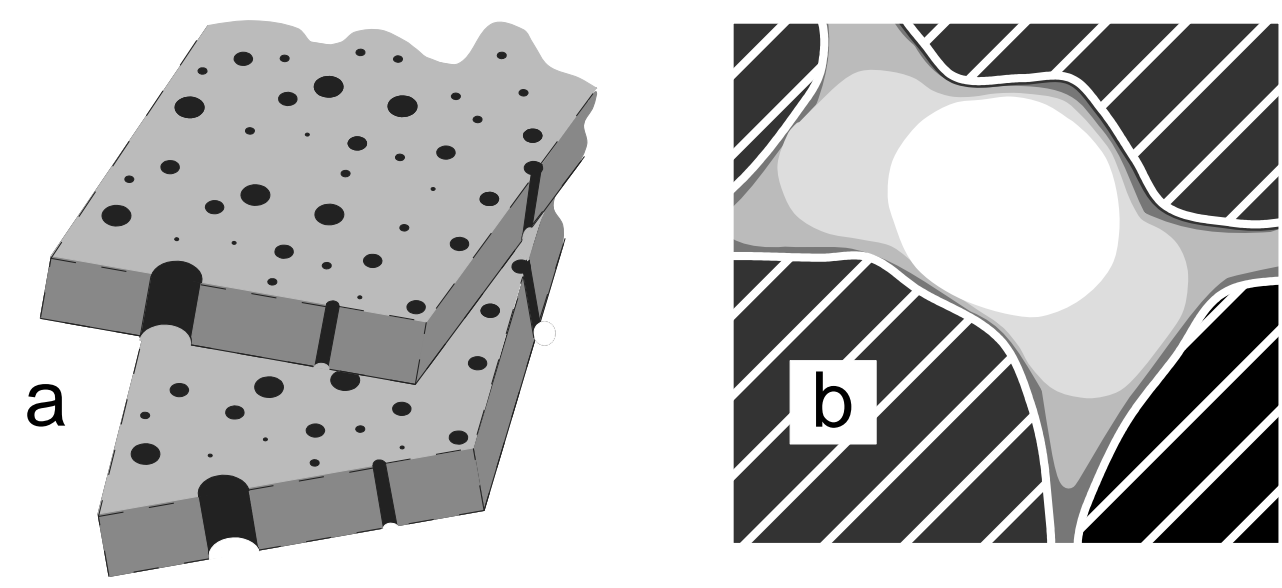

Fig. 1. Schematic visualization of two geometrical concepts for the pore space. (a) Pores of different sizes are spatially isolated holes in a solid matrix. The probability of pores of different sizes to be connected to each other is given by the product of their areal fraction at a cross section (concept of Childs and Collis-George (1953)). (b) The pore space desaturates gradually when the suction increases. The grey shadings represent different degrees of desaturation. Pores of similar "size" are in closer contact to each other than pores of very different sizes. 

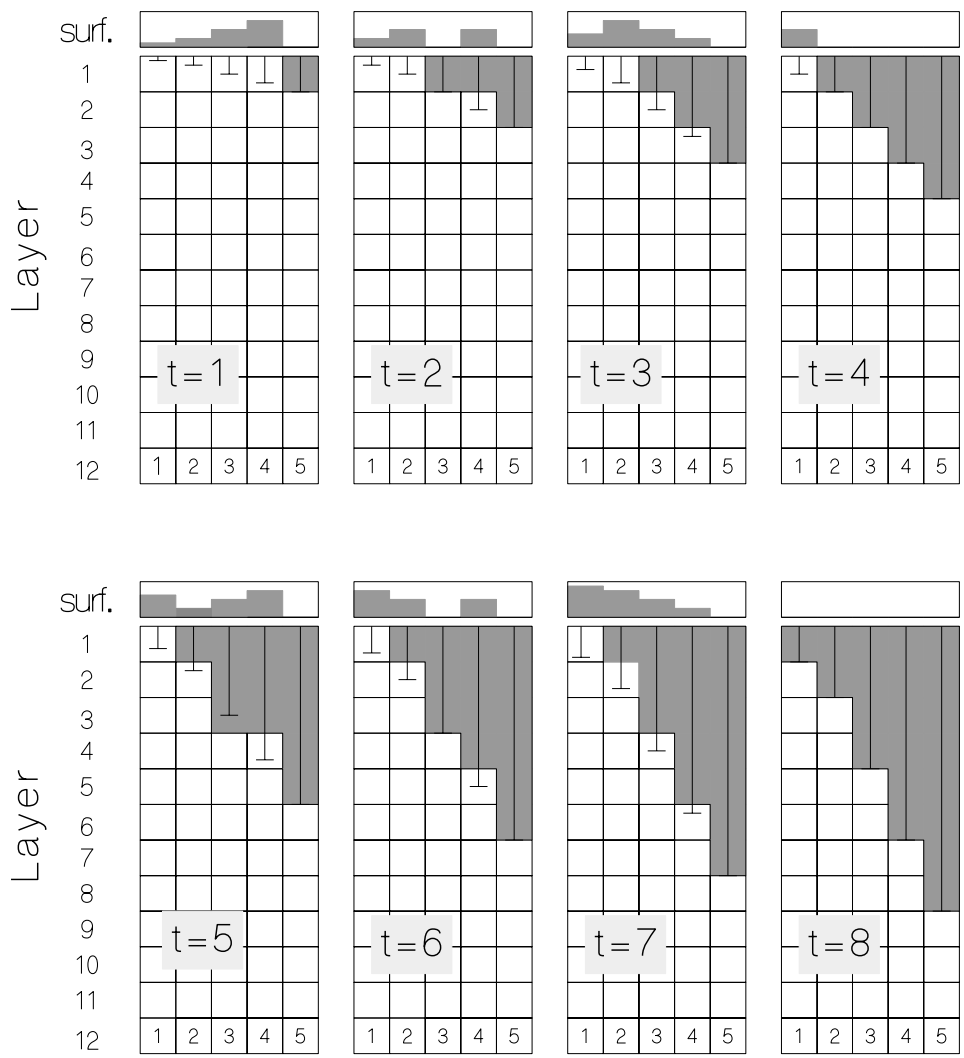

pore class

Fig. 2. Eight time steps of the propagation of a chemical into a water saturated soil. Each plot shows the cell arrangement for five pore classes (numbered in the lowest layer), and twelve depth layers. The relative velocities of the pore classes are $0.125,0.25,0.5,0.75$, and 1 , for pore classes 1 to 5 , respectively. White color indicates cells with concentration zero, grey shading indicates cells with relative concentration one. Exchange between the pore domains is zero (isolated tube model). The bars show the virtual propagation in each pore class. As soon as the virtual propagation is longer than the already propagated distance plus one layer thickness, a propagation step occurs. If no propagation occurs, the infiltrating chemical mass is stored in the surface layer (top of the cell array). 

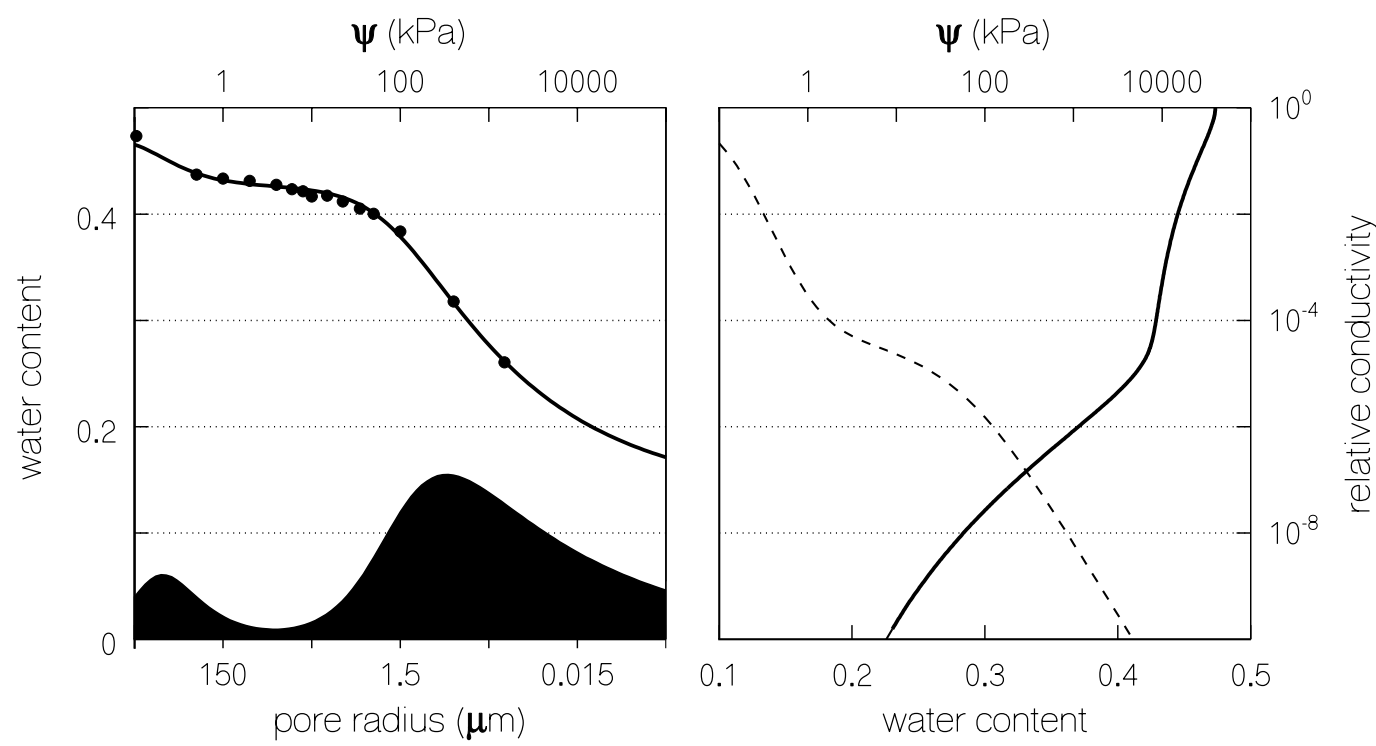

Fig. 3. Hydraulic properties of Rideau Clay Loam. Left: measured water retention data (dots), fitted bimodal retention curve (solid line), and underlying pore-size distribution (dark shaded, cf. Durner, 1994). Right: predicted functions $K(\psi)$ (dashed, related to top axis), and $K(\theta)$ (solid, related to bottom axis). 


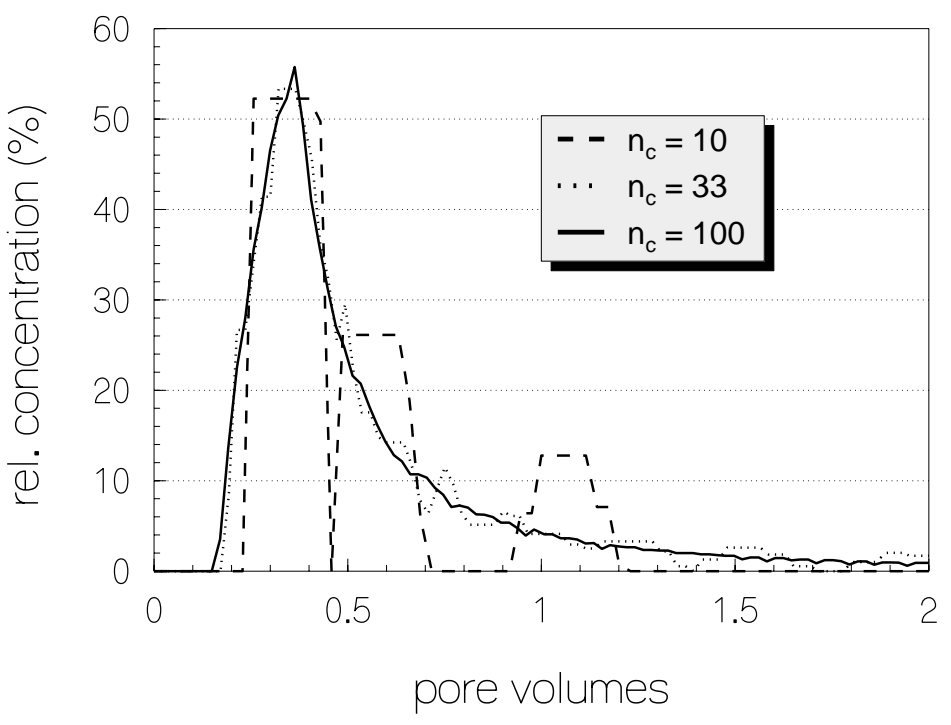

Fig. 4. Simulated steady-state breakthrough of a solute pulse of 0.2 pore volumes, applied to a $10 \mathrm{~cm}$ column of Rideau Clay Loam at $45 \%$ water content. Influence of varying the number of pore classes, $n_{c}$. No mixing between the pore domains is assumed 


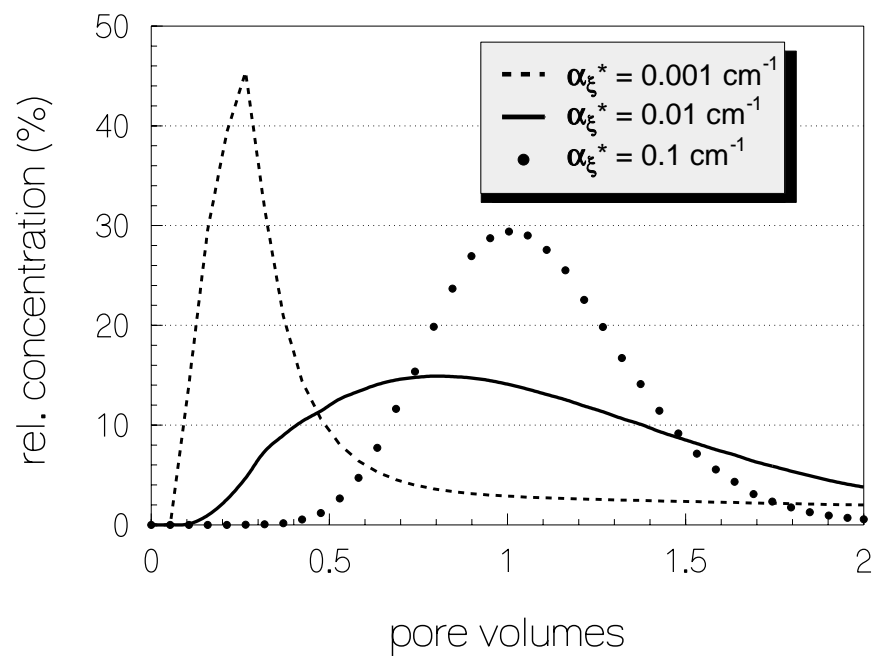

ref vardisp;file fig5

Fig. 5. Simulated steady-state breakthrough of a solute pulse of 0.2 pore volumes, applied to a $10 \mathrm{~cm}$ column of Rideau Clay Loam at $45 \%$ water content. Influence of varying the pore domain interaction coefficient, $\alpha_{\xi}$. 


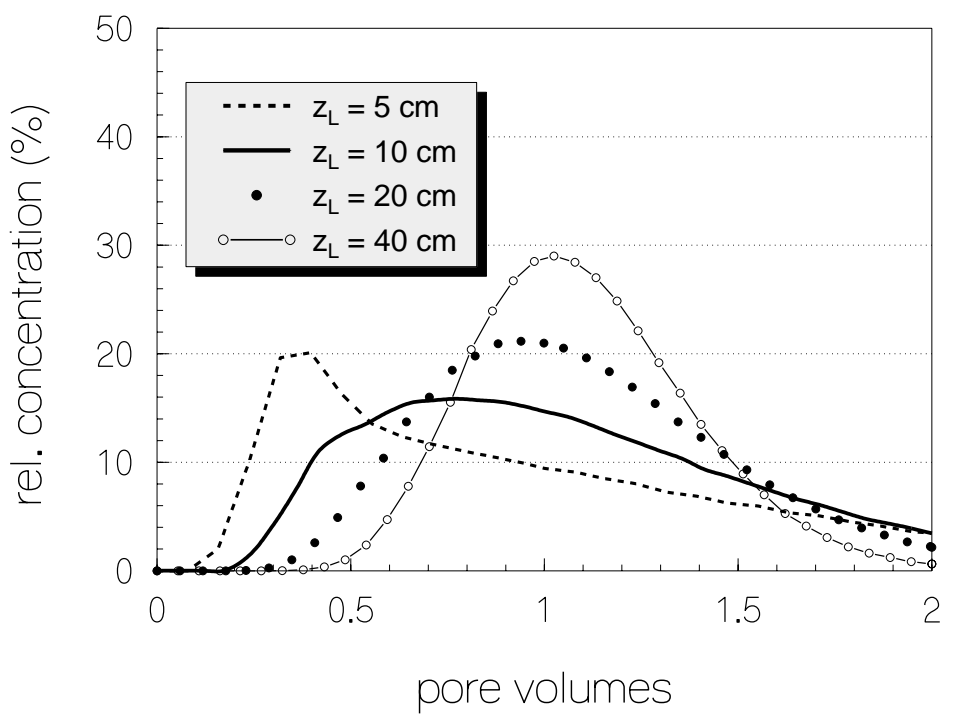

Fig. 6. Simulated breakthrough of a solute pulse of 0.2 pore volumes, applied to a column of Rideau Clay Loam at $45 \%$ water content. Normalized pore domain interaction factor is set to $\alpha_{\xi}^{*}=10^{-2}$. Influence of varying the column length. 


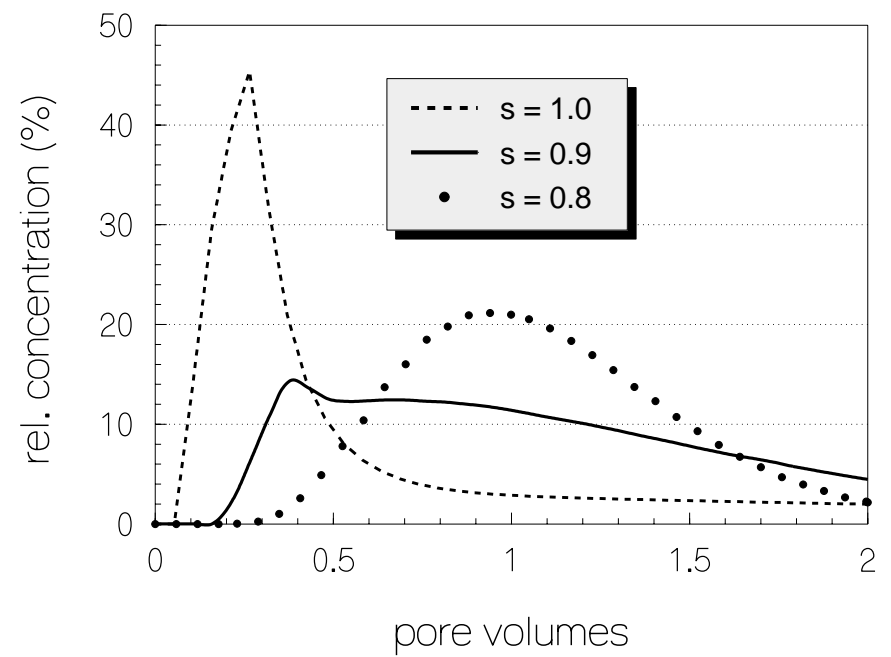

ref varsat;file fig7

Fig. 7. Simulated breakthrough of a solute pulse of 0.2 pore volumes, applied to a $10 \mathrm{~cm}$ column of Rideau Clay Loam. Normalized pore-domain interaction coefficient is set to $\alpha_{\xi}^{*}=$ $10^{-2}$. Influence of varying water saturation, $s$. 


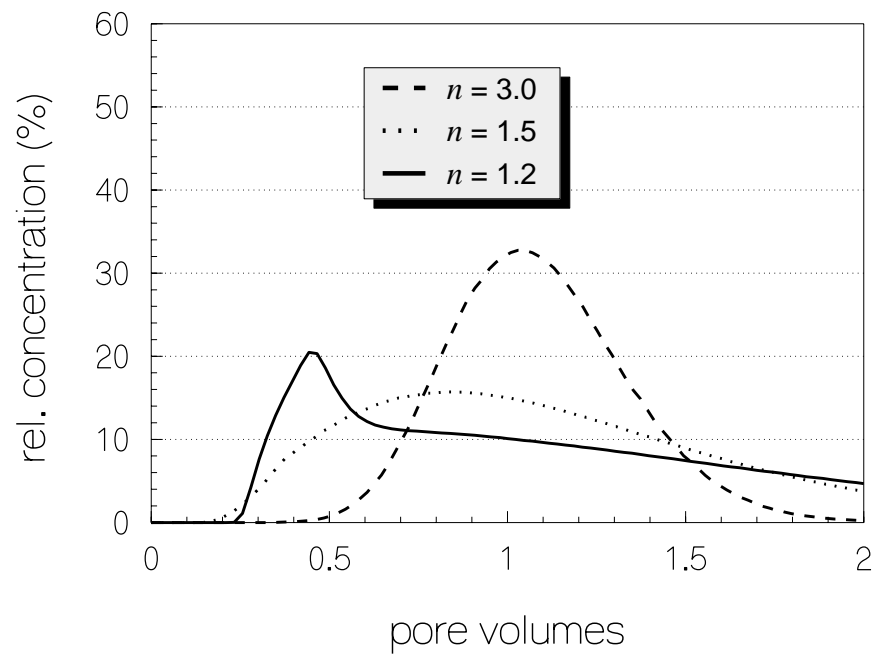

Fig. 8. Breakthrough curves through a saturated soil column of $20 \mathrm{~cm}$ length, comparing different pore-size distribution. The soil hydraulic properties are characterized by three different values of the parameter $n$ of the van Genuchten/Mualem hydraulic model (van Genuchten, 1980). Assumed $\alpha_{\xi}^{*}=10^{-2}$. 